\title{
CORRECTION
}

\section{Correction to: Focus on ARDS}

Ary Serpa Neto ${ }^{1,2}$, Armand Mekontso Dessap ${ }^{3,4}$ and Laurent Papazian ${ }^{5^{*}}$

C) 2017 Springer-Verlag GmbH Germany and ESICM

\section{Correction to: Intensive Care Med (2017)}

\section{3:1495-1497}

DOI 10.1007/s00134-017-4902-1

The second element of the second author's name was misinterpreted as a given name, whereas in fact it is part of his family name. The correct version of his name for indexing purposes is therefore A. Mekontso Dessap (not A.M. Dessap).

\section{Author details}

${ }^{1}$ Department of Critical Care Medicine, Hospital Israelita Albert Einstein, São Paulo, Brazil. ${ }^{2}$ Department of Intensive Care, Academic Medical Center, University of Amsterdam, Amsterdam, The Netherlands. ${ }^{3}$ Service de Réanimation Médicale, Assistance Publique - Hôpitaux de Paris, Hôpital Henri Mondor, DHU A-TVB, 94010 Créteil, France. ${ }^{4}$ Groupe de Recherche Clinique CARMAS, Faculté de Médecine, IMRB, Université Paris Est Créteil, 94010 Créteil, France. ${ }^{5}$ Réanimation des Détresses Respiratoires et Infections Sévères, Assistance Publique

- Hôpitaux de Marseille, Hôpital Nord, Aix-Marseille Université, Faculté de Médecine, 13015 Marseille, France.

Published online: 2 November 2017

\footnotetext{
${ }^{*}$ Correspondence: laurent.papazian@ap-hm.fr

${ }^{5}$ Réanimation des Détresses Respiratoires et Infections Sévères,

Assistance Publique - Hôpitaux de Marseille, Hôpital Nord, Aix-Marseille

Université, Faculté de Médecine, 13015 Marseille, France

Full author information is available at the end of the article
}

The online version of the original article can be found under doi:10.1007/s00134-017-4902-1.

\section{Springer}

